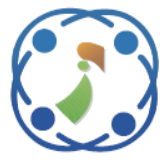

\title{
Improving the Efficiency of Water Management System in Biomass Power Plant Using Cyber-Physical Cloud Computing
}

\author{
Pranai Kungwalrut $^{1} \quad$ Viriya Kongratana $^{1} \quad$ Thanit Trisuwannawat $^{1}$ \\ Vittaya Tipsuwanporn ${ }^{1}$ Arjin Numsomran ${ }^{1 *}$ \\ ${ }^{I}$ Department of Instrumentation and Control Engineering, Faculty of Engineering, \\ King Mongkut's Institute of Technology Ladkrabang, Bangkok, Thailand \\ * Corresponding author's Email: arjin.nu@kmitl.ac.th
}

\begin{abstract}
Optimized water management strategies are among the most crucial concerns in a biomass power plant. Especially during the times of water scarcity, an improper water supply planning and operation result in a detrimental power generation or shut down processes. This paper aims to propose the cyber-physical cloud computing (CPCC), a mechanism of analysis and control physical process using cloud-based framework, in order to improve the efficiency of water management system in a biomass power plant. In this study, a 9.9 MW biomass power plant is implemented to investigate the performance of CPCC. The architecture of the proposed system consists of three physical tiers. The first tier is a tier of physical devices included with pressure, flow, $\mathrm{pH}$ sensors, water pumps and valves that are responsible for detecting the physical data and interacting with the water production process. The second tier is an edge computing device which functions as a controller, embedded server, data storage, gateway and switch to manage all operational tasks in an intranet area. The third tier is a cloud computing system which enables big data applications such as online monitoring and visualization of process operation, adaptive filtration fouling control, consuming water and total water cost analysis. The results validate the effectiveness of the proposed system as the ability of an adaptive fouling control system to adjust backwash scheduling and chemical dosing so that achieving the target of purified water quality even during the fluctuation of raw water qualities. Subsequently, the water treatment system can achieve the capability for optimal total water cost operations under a target permeate flow rate and the percent of the recovery. Significantly, the data analytics in the CPCC reflect current operational requirements of water under local climate conditions, likewise contribute to the practical solution for sustainable water resource planning in the energy production.
\end{abstract}

Keywords: Water management system, Biomass power plant, Cyber-physical cloud computing, Optimal energy consumption, Adaptive fouling control.

\section{Introduction}

Biomass power plant is one of the industries, which is highly dependent on water resources to complete the steam cycle and operate the electric turbines (Water for Energy 2010). Regarding Alternative Energy and Development Plan (AEDP), the Government of Thailand targets to increase biomass power plant capacity to 5,570 megawatts by the year 2036 [1]. The rising of biomass operation reflects huge volumes of water needs in the near future while many areas across the country are facing scarcity of fresh water due to climate changes and global warming [2]. Therefore, the effective water resource management is crucial issue for sustainable water and energy availability.

To accomplish a long-term availability of water reserved for power generation system, several solutions have been published. For instance, water treatment and reuse in a thermal power plant was proposed for providing the scenarios of reusing wastewater from power plant industries [3-4]. The water conservation management in thermal power 


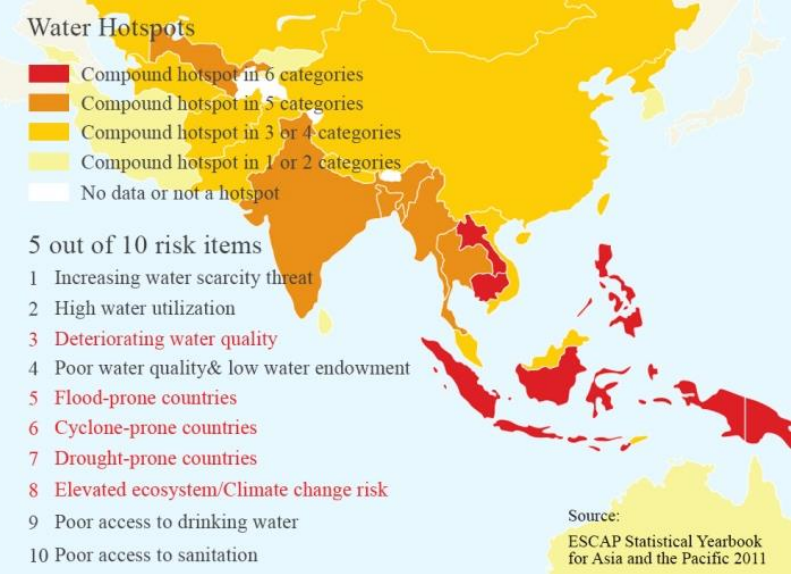

Figure.1 A potentially high water-risk country

plants was addressed with the intention of reducing the water consumption [5]. The modeling methods for estimating water consumption were proposed to analyze and predict maximum and minimum water consumption in the various types of thermo-electric power plants [6]. A low-water cooling technologies at thermos-electric power plants was addressed to evaluate drought resiliency using economic and technical performance parameters $[7,8]$.

Through the aforementioned aspects, this paper aims to propose the cyber-physical cloud computing (CPCC), a mechanism of analysis and control physical process using cloud-based framework, in order to improve the efficiency of water management system in a biomass power plant. In this study, a $9.9 \mathrm{MW}$ biomass power plant is implemented to investigate the performance of CPCC. Architecture of the proposed system consists of three physical tiers. The first tier is a tier of physical devices included with pressure, flow, $\mathrm{pH}$ sensors, water pumps and valves that are responsible for detecting the physical data and interacting with the water production process. The second tier is an edge computing device which functions as a controller, embedded server, data storage, gateway and switch to manage all operational tasks in an intranet area. The third tier is a cloud computing system which enables big data applications such as online monitoring and visualization of process operation, adaptive filtration fouling control, consuming water and total water cost analysis. The essential features advanced the previous methods are self-adaptive backwash and self-adaptive chemical dosing functioned for adaptive fouling control, likewise the feature of total water cost optimization which is used for controlling the energy consumption of water treatment system. The proposed method represents a valuable alternative to achieve the target of purified water quality even

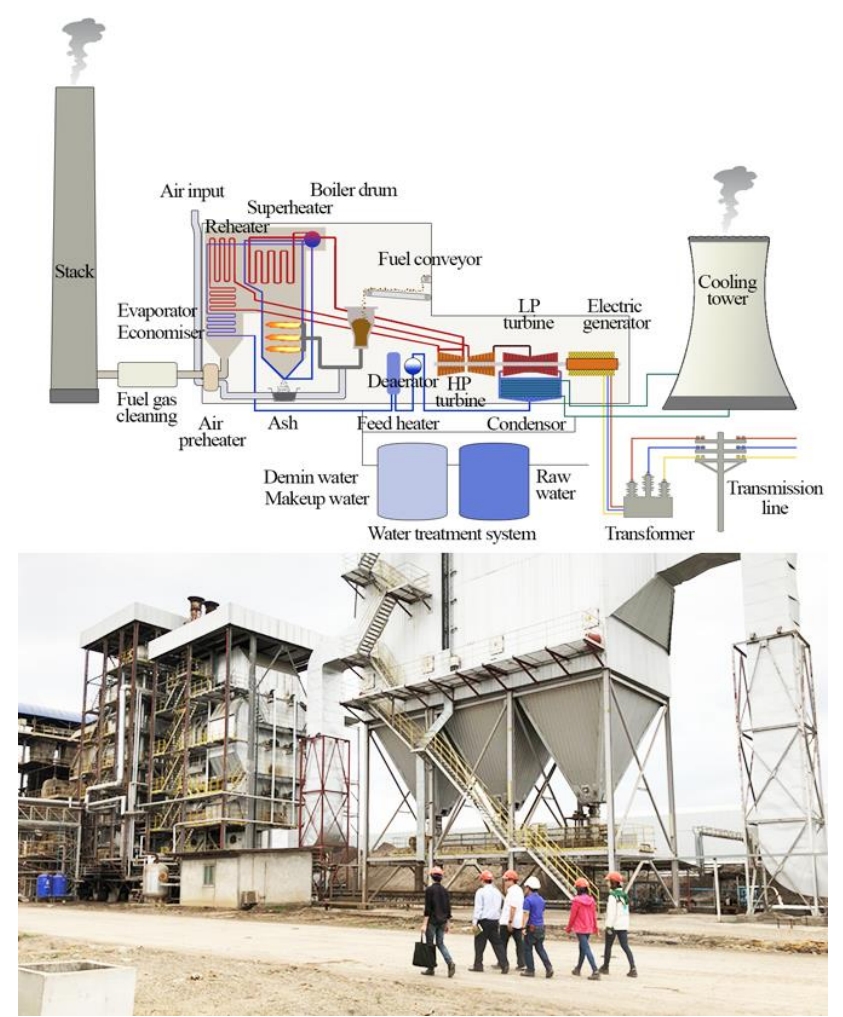

Figure. 2 Biomass power plant and schematic diagram

during the fluctuation of raw water qualities as well as increase the capability for optimal total water cost operations under a target permeate flow rate and the percent of the recovery. Significantly, more than the 12 months of the data analytics in the CPCC reflect current operational requirements of water under local climate conditions, achieve the superior performance of the water treatment operation, likewise contribute to strategic of sustainable water resource planning in the energy production. The rest of this paper is organized as follows: in section 2, Materials and methods of CPCC are described as a proposed system for water management system in a biomass power plant; in section 3 , the results obtained through experimental implementation has been evaluated; in section 4 , the discussion is presented; while section 5 is dedicated to the conclusions.

\section{Materials and methods}

\subsection{Biomass power plant}

The implemented biomass power plant site is located in Buriram, a province in the northeastern Thailand. It can generate $9.9 \mathrm{MW}$ of electricity production capacity using biomass fuel such as wood chips, bark, rice husk, corn cobs, corn stalk, wood shaving, sawdust and palm kernel shell from agricultural residues in the local area. 
Table 1. Biomass power plant description

\begin{tabular}{|l|c|}
\hline \multicolumn{1}{|c|}{ Description } & Value \\
\hline $\begin{array}{l}\text { Electrical energy output at full } \\
\text { condensing }\end{array}$ & $9.9 \mathrm{MW}$ \\
\hline Generator power factor & 0.85 \\
\hline Generator electrical voltage & $11,000 \mathrm{Volts}$ \\
\hline $\begin{array}{l}\text { Maximum steam flow at operating } \\
\text { pressure }\end{array}$ & $45 \mathrm{t} / \mathrm{hr}$ \\
\hline Operating pressure & $6.5 \mathrm{MPa}$ \\
\hline Operating temperature & $480^{\circ} \mathrm{C}$ \\
\hline $\begin{array}{l}\text { Feed water temperature at } \\
\text { economizer inlet }\end{array}$ & $110^{\circ} \mathrm{C}$ \\
\hline Saturated steam carryover moisture & $0.5 \%$ \\
\hline Fuel flow & $14.0 \mathrm{t} / \mathrm{hr}$ \\
\hline Particulate emissions & $<50 \mathrm{PPM}$ \\
\hline
\end{tabular}
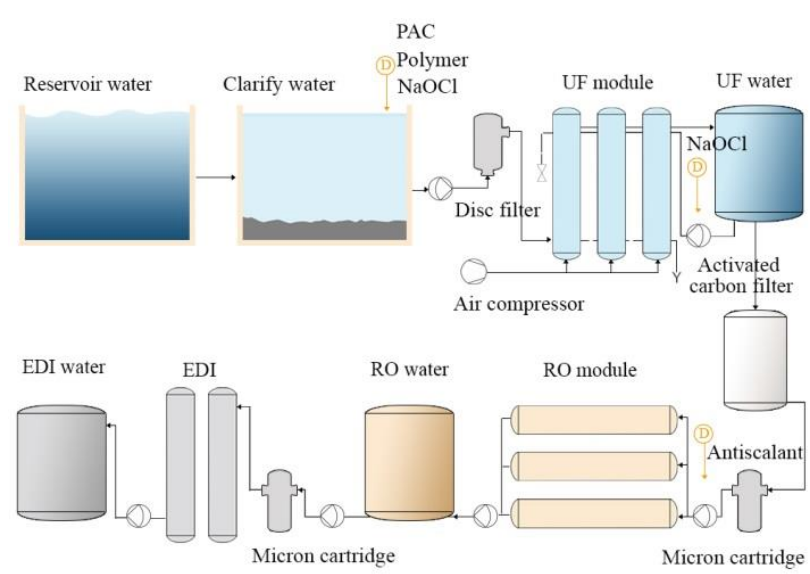

Figure.3 Schematic diagram of water treatment system

In Fig. 2, a schematic diagram demonstrates the structure of biomass power plant using a stoker boiler with a steam reheat system and extractioncondensing steam turbine for power generation [9]. The biomass fuel about 450 tons per day is burned in a direct-fired system of water tube boiler to create 45 tons per hour of high pressure steam. A system reuses the waste heat through preheater and reheater for increasing energy conversion efficiencies of almost $85 \%$. The open cycle cooling system composes of a steam, cooling tower, and cooling system pumps. The cooling water will be pumped from clarified water reservoir through the condenser and discharged back to the source. Air emissions are treated using electrostatic precipitators (ESP) for removing particulate matter. In addition, the details of biomass power plant description are mentioned in a Table 1.

\subsection{Water treatment system}

The water treatment process in a biomass power plant is divided by the purpose of using as a boiler water treatment and a cooling water treatment [1011]. The water treatment system of experimental biomass power plant is shown as the schematic diagram in Fig. 3. The system comprises of pretreatment, ultrafiltration, reverse osmosis and electrodeionization system that can purify water with low contaminants at flows up to $4 \mathrm{~m}^{3} /$ hour. The raw water from reservoirs is fed into a pre-treatment system for coagulation and disinfection using chemical dosing such as polyaluminium chloride, polymer and chlorine, and then the treated water is used as a clarified water in the cooling process. The remainder is fed into ultrafiltration and reverse osmosis system for $\mathrm{pH}$ adjusting and removing all the particles larger than 1.0 nanometer, and afterwards the water is treated in the electrodeionization system for eliminating ionizable species. The net output of $96 \mathrm{~m}^{3} /$ day high-purity permeate water from the last treatment stage is stored in a $120 \mathrm{~m}^{3}$ storage tank for preparation as a feed water in the steam production process. The characteristics of water treatment system are presented in Table 2.

To control the operation of water treatment system, currently the logical sequencing and timing control has been used by programmable logic controller [12]. However, using fixed timing of filtration or cleaning operation can induce the loss of membrane permeability, increase of operational expenditure, and reduction of treated water quality because the fluctuation characteristics of the raw water influenced by daily and seasonal variations $[13,14]$.

\subsection{Cyber-physical cloud computing (CPCC) technology for water management system}

The cyber-physical cloud computing (CPCC), a mechanism of analysis and control physical process using cloud-based framework, has been developed in this research with the aim of improving the efficiency of water management system in a biomass power plant. An infrastructure of the proposed system in Fig. 4 consists of three physical tiers: physical devices, edge computing device and cloud computing system [15].

\subsubsection{Physical devices}

The physical devices are the instruments included with pressure transmitter, temperature transmitter, flow transmitter, $\mathrm{pH}$ sensor, turbidity sensor, water pump and control valve that are responsible for detecting the physical data and interacting with the water production process. 


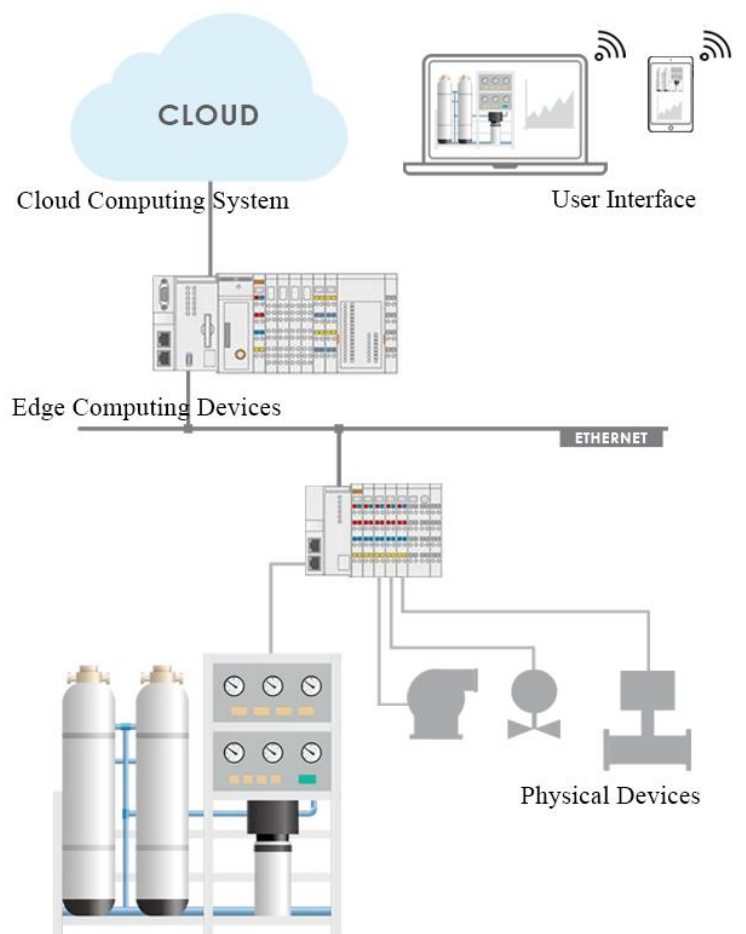

Figure.4 Cyber-physical cloud computing (CPCC) technology for water management system

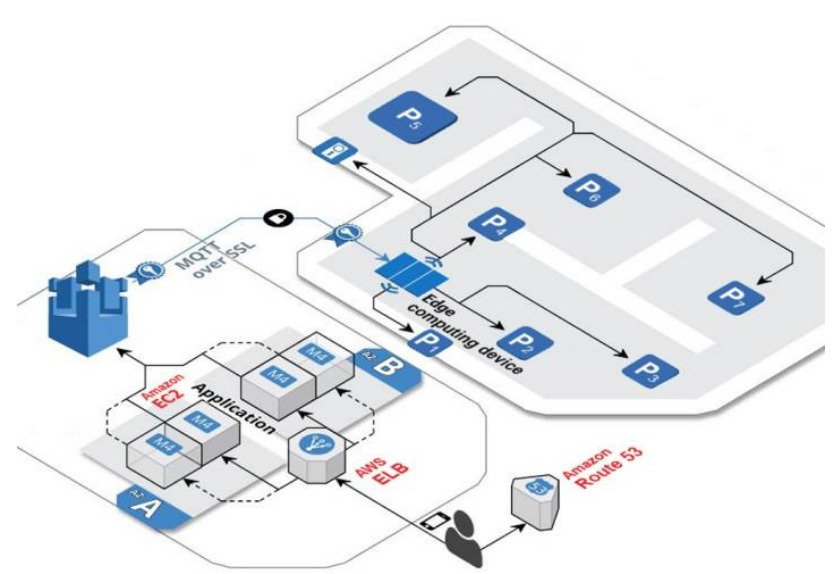

Figure.5 Cloud computing architecture

\subsubsection{Edge computing device}

The edge computing device is a programmable logic controller integrated with industrial IoT gateway which provides functionalities of process operation control as well as enable cloud connection to physical devices [16].

\subsubsection{Cloud computing system}

The cloud computing system is a platform of on-demand computing resources which provide the functionalities of data storage, online monitoring and visualization of process operation, process data processing and analysis application.

The proposed cyber-physical cloud computing technology for a water management system has a system architecture as demonstrated in Fig. 5. PLC edge computing device connects to remote $\mathrm{I} / \mathrm{O}$ of the physical device via Ethernet networks using MODBUS TCP protocol, so that controls water treatment process operation as well as collects and sends the specified data about once per hour to the cloud. The communication between edge computing device and public cloud platform is executed via MQTT protocol and encrypted via SSL 3.1. AWS IoT Core is a cloud management platform which supports communication protocol to enable secure connection including data transmission between physical devices and cloud services. Amazon EC2 provides secure and resizable computing resources implemented for developing the applications of adaptive filtration fouling control and consuming water and total water cost analysis. Amazon Route 53 provides scalable cloud Domain Name System (DNS) while AWS ELB is implemented to automatic load balancing. The operations data and analysis results are demonstrated in dashboard web service developed by JavaScript [17, 18].

\subsection{Total water cost}

In this research, the total water cost function is developed for assessment of operation cost by emphasizing the cost of chemical dosing and the cost of energy consumption.

\subsubsection{Chemical dosing cost}

Reagents in the water treatment system include $\mathrm{NaOCL}, \mathrm{NaOH}$, coagulant and antiscalant which can be is represented as the chemical dosing cost function by [19]

$$
f_{d o \sin g}=\left(\sum_{i=1}^{n} k_{i} u_{i}\right) Q_{r}
$$

$f_{\text {dosing }}$ : Total chemical reagent $\operatorname{cost}($ Baht $)$

$k_{i}$ : Unit price of reagent (Baht / unit)

$u_{i}$ : Dosage of reagent (unit/L)

$Q_{r}$ : Volume flow of raw water $(L / \mathrm{s})$

$f_{\text {dosing }}$ is a cost function representing the cost of all chemical dosing in water treatment plant which is proportional to the volume of applied reagents, the price of reagents and the volume of raw water. In this study, the total volume of chemical reagents have been measured and recorded for calculating the total water cost as well as using as a manipulate variable in self-adaptive chemical dosing process. 


\subsubsection{Energy consumption cost}

The energy consumption cost of water treatment system is expressed in this analysis as the Specific Energy Consumption (SEC), which is defined as the cost of the electrical energy required to purify a volume of permeate water [20-21].

$$
f_{S E C}=\frac{\left(\sum_{i} m_{i} Q_{f_{i}} \Delta P_{\text {pump }_{i}} / \eta_{\text {pump }_{i}}\right)}{Q_{\text {Ptotal }_{1} \Delta \pi_{0}}}
$$

$f_{S E C}$ : Total energy comsumption cost $($ Baht $)$ $m_{i}$ : Unit price factor $(B a h t / k W \cdot h)$ $Q_{f_{i}}$ : Volume flow rate offeed water $\left(\mathrm{m}^{3} / \mathrm{h}\right)$ $\Delta P_{\text {pump }}$ : Difference pressure across pump (Bar) $\eta_{\text {pump }}:$ Pump efficiency

$Q_{P \text { total }}:$ Total flow rate ofpermeate $\left(\mathrm{m}^{3} / \mathrm{h}\right)$ $\Delta \pi_{0}$ : Osmotic pressure (bar)

$f_{S E C}$ is a cost function representing the cost of energy consumption to purify the water in a RO water treatment plant which is mainly proportional to the values of feed flow rate $\left(Q_{f}\right)$, differential pressure across the pump $\left(\Delta P_{\text {pump }}\right)$, permeate flow rate $\left(Q_{P}\right)$. In this study, there are five control variables (the feed pressure, feed flow rate, concentrate pressure, concentrate flow rate and water recovery) which the optimal values are determined. The optimization objective is to minimize the energy consumption that affects to the total water cost, while producing a specified volume of permeate water.

\subsection{Optimization of energy consumption}

The optimization of energy consumption in water treatment operation is developed using the energy-optimal control system which control feed pressure and flow rates of $\mathrm{RO}$ system for achieving the water treatment recovery at the energy optimal conditions [22]. The schematic diagram of energyoptimal control system in Fig. 6 demonstrates the cooperation between controllers and RO water treatment system. The two stages of RO water treatment system are controlled by an edge controller for achieving the required permeate flow rate set-point at the specified water recovery, while the supervisory controller calculates the optimal RO feed pressure and the feed flow rate so that establish the trajectories and operational set-point for edge computing control. The actuators are high pressure pump and concentrate valve which are used for adjusting RO feed pressure, feed flow rate and concentrate flow.

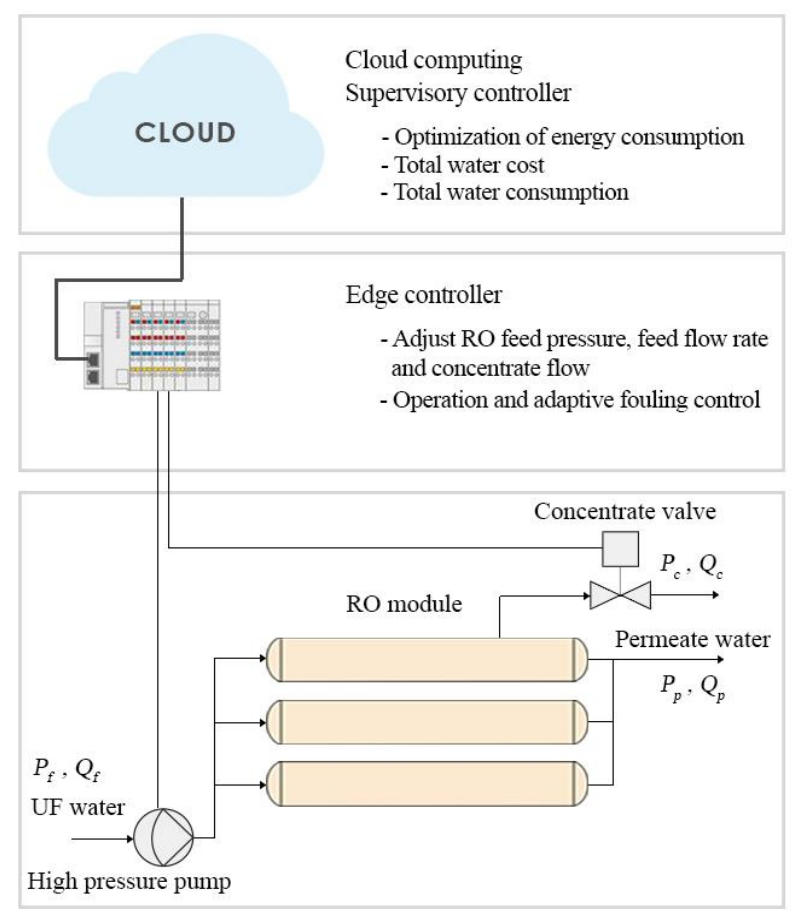

Figure.6 Energy-optimal control system

\subsection{Adaptive fouling control methodology}

The actual applications of membrane water treatment system are still confronted with the problem of filtration and fouling control because the fluctuation characteristics of the raw water influenced by daily and seasonal variations. An improper water treatment process operation results in the loss of membrane permeability, increase of operational expenditure, and reduction of treated water quality. Therefore, an adaptive fouling control methodology is developed as a crucial part of cyber physical cloud computing for optimizing water treatment operation in the appropriate scheduling and chemical dosing quantity in order to treat the varying quality of raw water efficiently [23-24].

\subsubsection{UF self-adaptive backwash scheduling}

This research develops the self-adaptive backwash scheduling using a deviation of UF resistance as a threshold triggering for air scouring backwash [25].

$$
R_{T}=\frac{\Delta P_{m}}{\mu \cdot J}
$$

where

$$
J=\frac{n \cdot Q_{U F}}{A_{m}}
$$




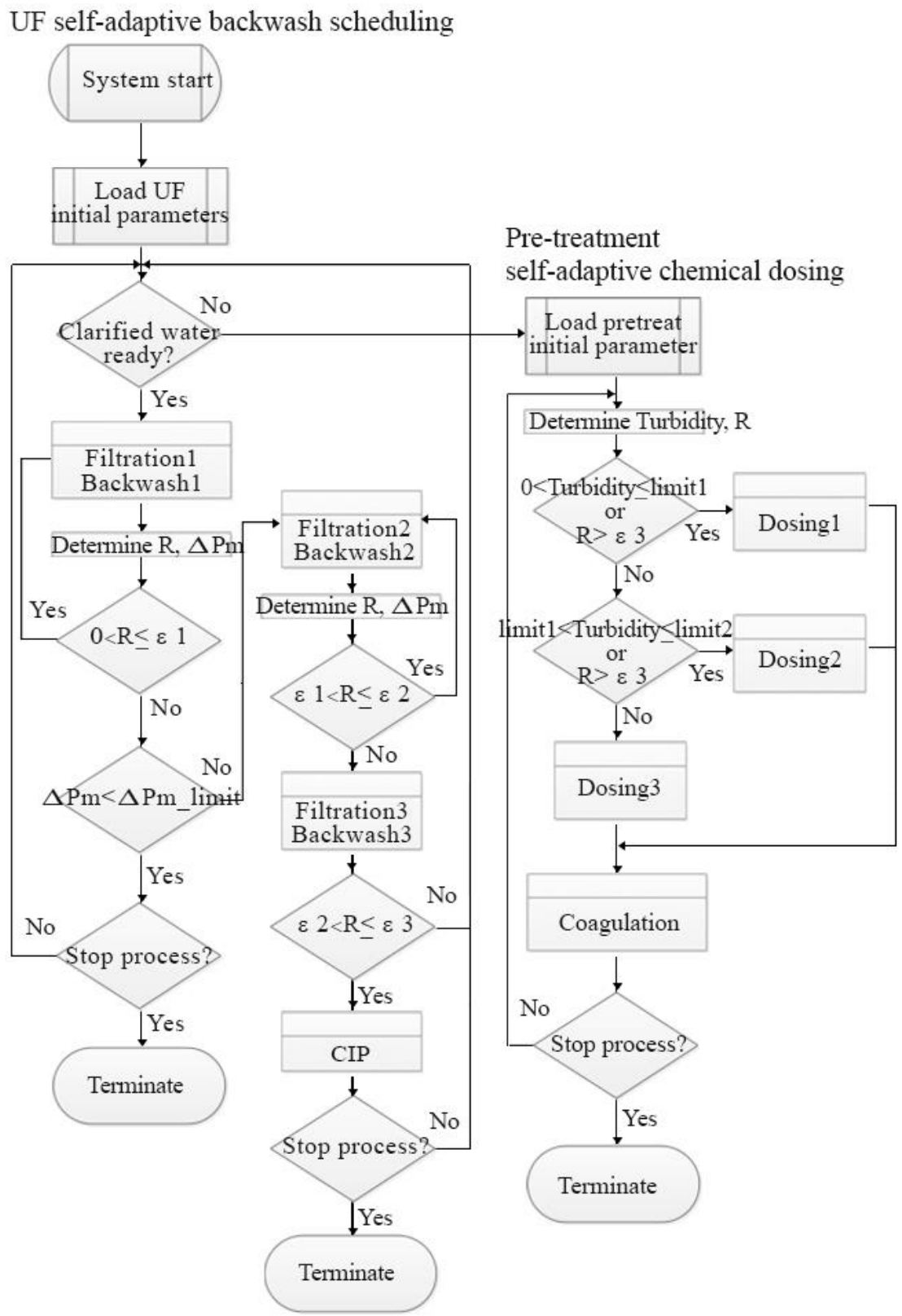

Figure.7 A flow diagram of the adaptive fouling control

$R_{T}$ is the total UF resistance. $\Delta P_{m}$ is pressure difference across the membrane (Bar). $\mu$ is the water viscosity (mPa.s). $J$ is penetration flux $\left(\mathrm{L} / \mathrm{m}^{2} \cdot \mathrm{h}\right) . n$ is number of UF skids. $Q_{U F}$ is permeate flow rate of the UF skid. $A_{m}$ is the active membrane area.

In self-adaptive backwash control, the 1'st filtration and backwash scheduling plan is performed an operation as an initial state. In case the value of UF resistance $\left(R_{T}\right)$ rises towards the threshold ( $\varepsilon 1, \varepsilon 2, \varepsilon 3)$, the adaptive fouling control is proceeded by adjusting the filtration and backwash scheduling plan which properly with varying characteristics of the raw water.

\subsubsection{Pre-treatment self-adaptive chemical dosing}

A pre-treatment is an important part of water treatment system, functioning as a first contamination screening. Numerous chemicals are used for coagulant and disinfection. An improper quantity of chemical dosing results in the increasing of operational expenditure. Therefore, self-adaptive chemical dosing is developed in this research.

The self-adaptive chemical dosing controller collects physical data of raw water, such as $\mathrm{pH}$, turbidity and calculates the value of UF resistance so that performs the effective chemical dosing control corresponding to the presence of substances in raw water along with UF resistance. 
The self-adaptive fouling control algorithm of water treatment system for backwash scheduling and chemical dosing is demonstrated in Fig. 7.

\section{Experimental results}

As aforementioned research methodology, the experiments of proposed cyber-physical cloud computing (CPCC) were implemented on a water treatment system of biomass power plant in Fig. 3 to validate system efficiency. The experiment tests included performance validation of water treatment system, particularly the ability of an adaptive fouling control system to adjust backwash scheduling and chemical dosing complying with the varying properties of raw water. Subsequently, the capability of total water cost optimization in biomass power plant compared with existing operation was considered.

Referring to the membrane resistance previously mentioned in Eq. (3), $R_{T}$ has been calculated and used as a threshold triggering for air scouring backwash so that decreases the membrane fouling rate as well as increases the purification performance. The UF controller operated filtrationbackwash cycles with adaptive backwash scheduling. The 1'st filtration and backwash scheduling plan was performed an operation as an initial state. In case the value of UF resistance $\left(R_{T}\right)$ rose towards the thresholds $\left(\varepsilon_{1}=1.5, \varepsilon_{2}=2.5, \varepsilon_{3}=10\right)$, the appropriate scheduling plan was operated with air-pressure entrance pressure 2.5 bar and flow rate $12 \mathrm{~m}^{3} / \mathrm{hr}$ for 20, 40 and 60 second interval time. The system produced the filtrate flux of $100 \mathrm{~L} / \mathrm{m}^{2} / \mathrm{hr}$ at feed pressure 3 bar. UF membrane resistances resulting from self-adaptive backwash scheduling control, compared with fix backwash scheduling are illustrated in Fig. 8. It is noted that the proposed self-adaptive backwash scheduling impacts on the lower rate of UF resistance increase (Blue line) compared to the fixed frequency backwash (Red line). The result demonstrated that self-adaptive backwash, based on a membrane resistance threshold, was superior to fixed frequency backwash in decreasing the membrane fouling rate and extending the frequency of requiring chemical cleaning in place (CIP), likewise increases the effectiveness of water purification [26].

Pre-treatment controller operated the water clarifying cycles with adaptive chemical dosing. The raw water turbidity and UF membrane resistances mentioned in Eq. (3) were considered for controlling the chemical dosing quantity corresponding to the quality of raw water. The system produced the clarify water of $100 \mathrm{~m} 3 / \mathrm{hr}$. Adaptive chemical dosing manipulation corresponding to raw water turbidity was performed using different chemical reagents volume $3.5,5.5,8 \mathrm{~L} / \mathrm{s}$ complied by respectively water turbidity limit 20, 25, 30 NTU. The results in Fig. 9 demonstrated that the proposed self-adaptive chemical dosing achieved the effectiveness of adaptive fouling control compared to conventional water treatment processes which always applies fixed volume of chemical regents affects the worse cost of exceeded chemical dosing or lower purified water quality [27]. The adjustable chemical dosing volume (Yellow line) is capable of change corresponding to the presence of raw water turbidity (Blue line) so that decreasing membrane fouling rate of filtration membrane represented by the UF resistance (Red line) even in the situation of high suspended particulates in the raw water.

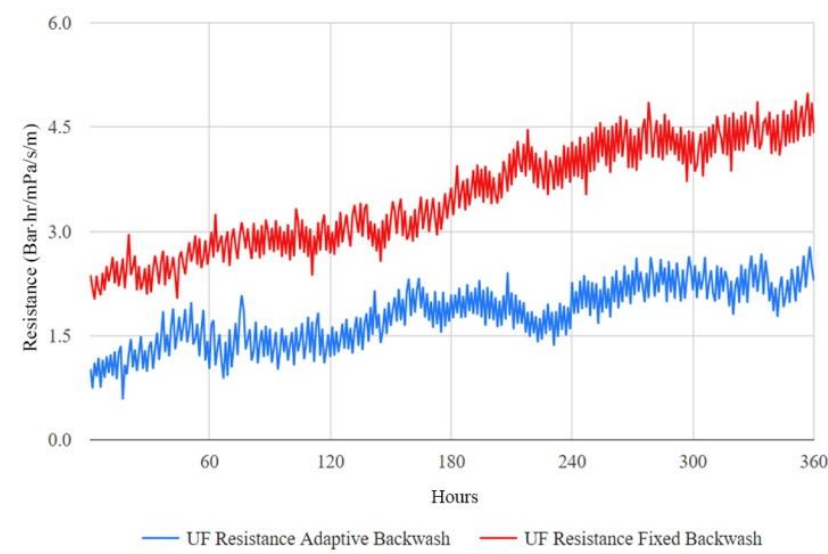

Figure.8 UF membrane resistance with self-adaptive backwash

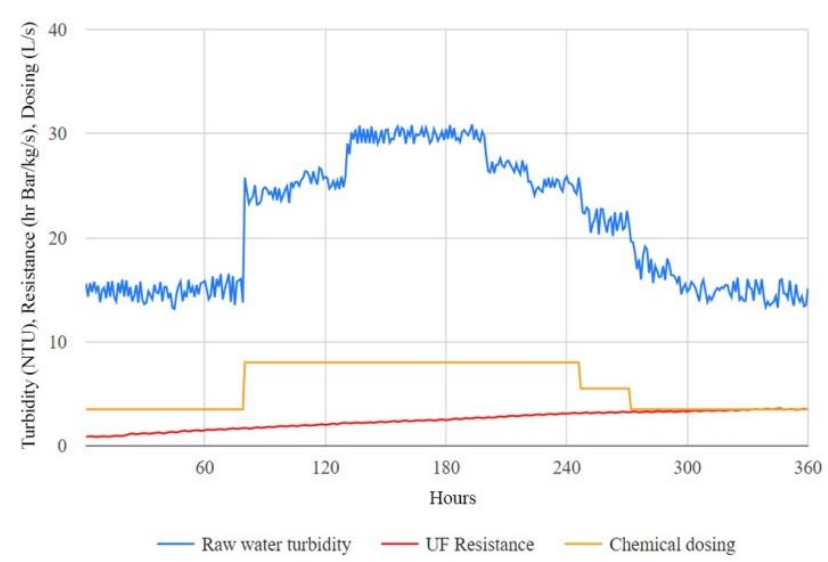

Figure.9 Adaptive fouling control impact

RO water treatment operation constrained by target recovery $(Y)$, required permeate flow rate $\left(Q_{p}\right)$, feed flow rate $\left(Q_{f}\right)$, maximum feed pressure $\left(P_{f}\right)$ and permeate pressure $\left(P_{p}\right)$. Low-level RO filtration controller received set point of RO feed pressure and concentrate flow from the supervisory RO controller as well as collected process variables $\left(P_{f}, Q_{f}, P_{c}, Q_{c}\right.$, 
$\left.P_{p}, Q_{p}, \quad\right)$ so that performed system operation maintain energy optimal as derived in Section 2.4.2. Referring to given permeate flow rate $\left(Q_{p}\right)$ of $4 \mathrm{~m}^{3} / \mathrm{h}$, maximum permeate pressure $\left(P_{f}\right)$ of $8.5 \mathrm{Bar}$, and recovery $(Y)$ of $90 \%$ will lead to calculate the set point of RO feed pressure $\left(P_{f}\right)$ and feed flow rate $\left(Q_{f}\right)$ that is relative to the optimal energy consumption cost function expressed by Eq. (2).

As aforementioned methodology, water treatment system is able to achieve the target of improving the operation efficiency as illustrated by the impact on total water cost in Fig. 10 as well as purified water quality in Fig. 11. The results in Fig. 10 demonstrated the total water cost (Blue line) of water treatment operation for biomass power plant which computed by the summation of chemical dosing cost in Eq. (1) and energy consumption cost in Eq. (2) added with the average fixed cost (Yellow line). Comparing with the average total water cost of the system without the proposed cyber-physical cloud computing (Red line) [28], the total water cost (Blue line) was decreased by the major impact of chemical dosing cost reduction as well as energy consumption cost optimization. Furthermore, the results in Fig. 11 illustrated the fact that the water treatment operation with the functions of proposed adaptive-fouling control and energy consumption optimization was able to achieve the effectiveness of water purification complied by the required specification EDI TDS $<0.64$ (Orange area), EDI $\mathrm{pH}=6.5-8.5$ (Orange line) UF/RO TDS $<6.5$ (Blue area) and UF/RO $\mathrm{pH}=5.5-7.5$ (Blue line), even during the fluctuation of raw water qualities.

In this research, the total water consumption has been observed and demonstrated as the graphical data depicted in Fig. 12 which is the important information from the proposed system reflecting the operational requirement of water under local climate conditions. Comparing with the conventional biomass power plant [29-30], the proposed cyberphysical cloud computing (CPCC) is able to expose the crucial data which are appropriately used for water resources planning in case of water scarcity.

\section{Discussion}

The performance of CPCC for water management system was initially concentrated on capability for adaptive fouling control corresponding to the variation of raw water properties. In Fig. 8, the comparison of fouling control results, UF membrane resistance with selfadaptive backwash and fixed backwash are demonstrated. In case higher of turbidity of raw water and pressure across the membrane, backwash

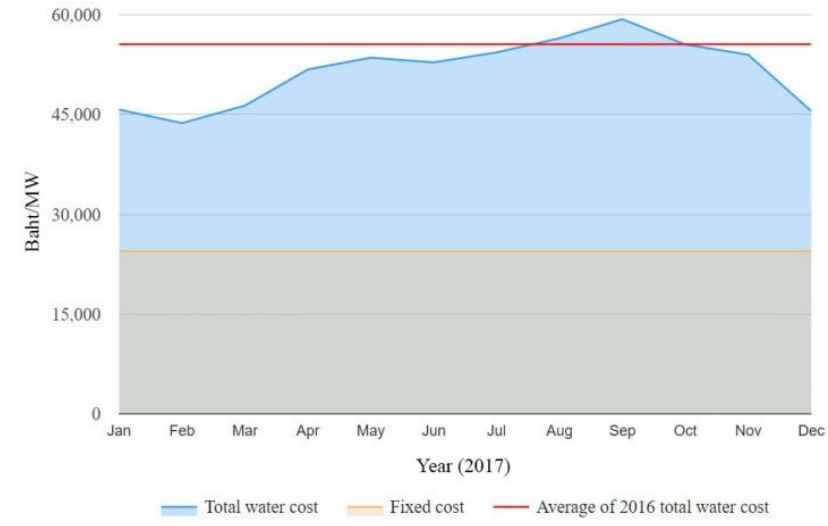

Figure.10 Total water cost

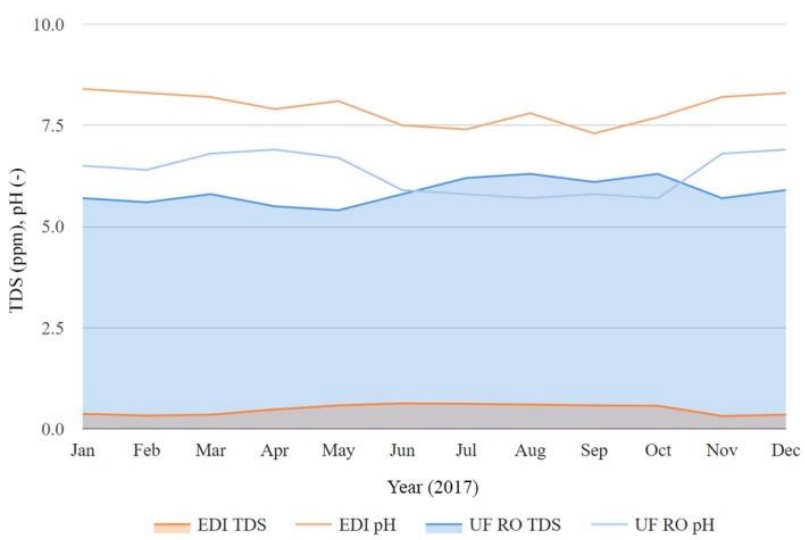

Figure.11 Water treatment quality

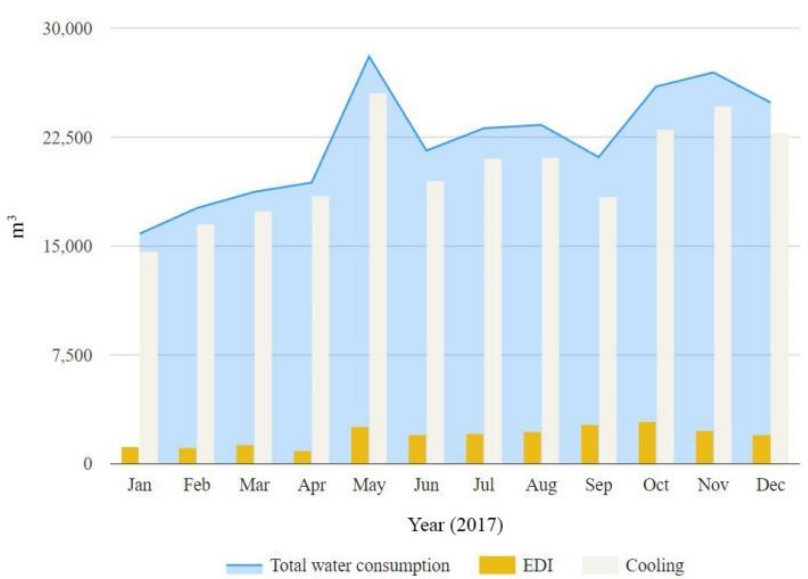

Figure.12 Total water consumption

frequency and interval time was increased, while filtration interval time was decreased so that decreased raising the rate of membrane resistance. In Fig. 9, self-adaptive chemical dosing control was illustrated. The different dosing plans were operated corresponding to variation of raw water turbidity. The results of self-adaptive fouling in Fig. 10, demonstrate that the control action can achieve the target to control, purified water quality complied by the required specification TDS $<0.64, \mathrm{pH}=6.5-8.5$, even during the fluctuation of raw water qualities, 
furthermore can restrain a membrane fouling as well as extend a membrane lifetime. Subsequently, the capability of optimal energy operation was focused. In Fig. 11 clearly presents that the water treatment system can achieve the capability for optimal total water cost operation under a target permeate flow rate $4 \mathrm{~m}^{3} / \mathrm{h}$ and recovery $(Y)$ of $90 \%$. In this research, the total water consumption has been observed as the graphical data depicted in Fig. 12 which is the appropriate information reflecting the operational requirement of water under local climate conditions, also it is available for a water resource planning in the energy production.

\section{Conclusion}

Improving the efficiency of water management system in a biomass power plant using cyberphysical cloud computing has been presented in this study. The proposed infrastructure and methodologies were developed and implemented on
$\mathrm{UF} / \mathrm{RO}$ water treatment process of biomass power plant. The three physical tiers constructed of physical instrumentation, edge computing and cloud computing were deployed with the aim of providing the superior performance of the water treatment operation. The self-adaptive fouling control efficiently accomplished a capability of purification during variation of raw water properties. In case of optimal total water cost operation, the energy consumption and chemical dosing were optimized while achieving the requirement of permeates flow rate and percent of the recovery. Furthermore, the tracking of total water consumption reflected the volumes of water needs which advantaged for a water resource planning in the energy production.

The future research plans include optimization of fuel consumption and fuel cost of biomass power generation for improving combustion efficiency, likewise sustaining operation resources.

Table 2. Characteristics of water treatment system

\begin{tabular}{|l|c|c|c|c|c|}
\hline Water quality parameter & Units & Raw water & Pre-Treatment & UF/RO & EDI \\
\hline TDS & $\mathrm{mg} / \mathrm{L}$ & 1920 & 1984 & $<6.4$ & $<0.64$ \\
\hline $\mathrm{pH}$ & - & 7.25 & 7.2 & $5.0-9.0$ & $6.5-8.5$ \\
\hline Turbidity & $\mathrm{NTU}$ & 15.68 & 1 & $<0.1$ & $<0.1$ \\
\hline
\end{tabular}

Nomenclature

\begin{tabular}{|l|l|l|l|}
\hline$P_{f}$ & Pressure of feed water (Bar) & $\Delta P_{\text {pump }}$ & Difference pressure across pump (Bar) \\
\hline$P_{c}$ & Pressure of concentrate (Bar) & $\Delta P_{m}$ & Pressure across membrane $(\mathrm{Bar})$ \\
\hline$P_{P}$ & Pressure of permeate $(\mathrm{Bar})$ & $Y$ & RO Recovery rate $(\%)$ \\
\hline$Q_{f}$ & Flow rate of feed water $\left(\mathrm{m}^{3} / \mathrm{h}\right)$ & $R_{T}$ & Total UF resistance $(\mathrm{Bar} / \mathrm{mPa} . \mathrm{s} \cdot \mathrm{m} / \mathrm{hr})$ \\
\hline$Q_{c}$ & Flow rate of concentrate $\left(\mathrm{m}^{3} / \mathrm{h}\right)$ & $n$ & Number of UF skids \\
\hline$Q_{P}$ & Flow rate of permeate $\left(\mathrm{m}^{3} / \mathrm{h}\right)$ & $A_{m}$ & Active membrane area $\left(\mathrm{m}^{2}\right)$ \\
\hline$Q_{P \text { total }}$ & Total flow rate of permeate $\left(\mathrm{m}^{3} / \mathrm{h}\right)$ & $\mu$ & Dynamic viscosity $(\mathrm{Pa} . \mathrm{s})$ \\
\hline$Q_{U F}$ & Total flow rate of UF permeate $\left(\mathrm{m}^{3} / \mathrm{h}\right)$ & $\varepsilon$ & Maximum threshold of UF resistance \\
\hline$\eta_{\text {pump }}$ & Pump efficiency & $k_{i}$ & Unit price of reagent $(\mathrm{Baht} / \mathrm{Unit})$ \\
\hline$\Delta \pi_{0}$ & Osmotic pressure (bar) & $m_{i}$ & Unit price of energy (Baht/Unit) \\
\hline
\end{tabular}

\section{References}

[1] P. Gadonneix, "Water for Energy", World Energy Council, 2010.

[2] Ministry of Energy, "Alternative Energy and Development Plan (AEDP) 2015-2036", THAILAND, 2015.

[3] T. Koanantakool, "Thai Energy Policy for Science and Technology Development", The $4^{\text {th }}$ Kyoto University Asian Network Forum, Japan, 2016.
[4] B. Al Smadi, K. Alzboon, and T. Al Azab, "Water Management and Reuse Opportunities in a Thermal Power Plant in Jordan", African Journal of Biotechnology, Vol. 9, No. 29, pp. 4606-4614, 2010.

[5] A. Mercè Rio Carrillo and C. Frei, "Water: A Key Resource in Energy Production", Energy Policy, Vol. 37, No. 11, pp. 4303-4312, 2009.

[6] M. S. Mohsen, "Treatment and Reuse of Industrial Effluents: Case Study of a Thermal Power Plant", Desalination, Vol. 167, pp 75-86, 2004. 
[7] J. M. Salazar, U, Diwekar, E. Constantinescu, and V. M. Zavala, "Stochastic Optimization Approach to Water Management in CoolingConstrained Power Plants", Applied Energy, Vol. 112, pp. 12-22, 2013.

[8] M. Farahani, S. M. Borghei, and V. Vatanpour, "Recovery of Cooling Tower Blowdown Water for Reuse: The Investigation of Different Types of Pretreatment Prior Nanofiltration and Reverse Osmosis", Journal of Water Process Engineering, Vol. 10, pp. 188-199, 2016.

[9] K. J. Ptasinski, "Efficiency of Biomass Energy", John Wiley \& Sons, Inc., 2016.

[10] J. Lee, K. Park, H. Eum, and C. Lee, "Desalination of a Thermal Power Plant Wastewater by Membrane Capacitive Deionization", Desalination, Vol. 196, No. 1-3, pp. 125-134, 2006.

[11] M. Xing, "Study on the Water Conservation Management Measures in Thermal Power Plants", International Journal of Business and Management, Vol. 5, No. 3, pp. 160-164, 2010.

[12] L. O. Villacorte, S. Tabatabai, D. M. Anderson, G. L. Amy, J. C. Schippers, and M. D. Kennedy, "Seawater Reverse Osmosis Desalination and (Harmful) Algal Blooms", Desalination, Vol. 360, pp. 61-80, 2015.

[13] L. Henthorne and B. Boysen, "State of the Art of Reverse Osmosis Desalination Pretreatment", Desalination, Vol. 356, pp. 129-139, 2015.

[14] X. Zheng, S. Plume, M. Ernst, J. Croué, and M. Jekel, "In-Line Coagulation Prior to UF of Treated Domestic Wastewater - Foulants Removal, Fouling Control and Phosphorus Removal", Journal of Membrane Science, Vol. 403-404, pp. 129-139, 2012.

[15] C. Kirsch, E. Pereira, and R. Sengupta, "CyberPhysical Cloud Computing: The Binding and Migration Problem", In: Proc. of the International Conf. on Design, Automation \& Test in Europe, pp. 1425-1428, 2012.

[16] K. Bilal, O. Khalid, A. Erbad, and S. U. Khan, "Potentials, Trends, and Prospects in Edge Technologies: Fog, Cloudlet, Mobile Edge, and Micro Data Centers", Computer Networks, Vol. 130, pp. 94-120, 2018.

[17] X. F. Liu, M. Shahriar, S.M. N. Al Sunny, M. C. Leu, and L. Hu, "Cyber-Physical Manufacturing Cloud: Architecture, Virtualization, Communication, and Testbed", Journal of Manufacturing Systems, Vol. 43, No. 2, pp. 352364, 2017.

[18] B. Varghese and R. Buyya, "Next Generation Cloud Computing: New Trends and Research
Directions", Future Generation Computer Systems, Vol.79, No. 3, pp. 849-861, 2018.

[19] T. Zhou and Z. W. Li, "A Cost Model Approach for RO Water Treatment of Power Plant", Procedia Environmental Sciences, Vol. 11, pp. 581-588, 2011.

[20] G. K. Pearce, "UF/MF Membrane Water Treatment Principle and Design", Water Treatment Academy, TechnoBiz Communications Co., Ltd., 2012.

[21] N. Zarai, F. Tadeo, and M. Chaabene, "Planning of the Operating Points in Desalination Plants Based on Energy Optimization", International Journal of Computer Applications, Vol. 68, No. 18, pp. 611, 2013.

[22] M. Li, "Optimization of Multitrain Brackish Water Reverse Osmosis (BWRO) Desalination", Industrial \& Engineering Chemistry Research, Vol. 51, No. 9, pp. 3732-3739, 2012.

[23] T. Nguyen, F. Roddick, and L. Fan, "Biofouling of Water Treatment Membranes: A Review of the Underlying Causes, Monitoring Techniques and Control Measures", Membranes, Vol. 2, No. 4, pp. 804-840, 2012.

[24] L.G. van Willigenburg, H.M. Vollebregt, and R.G.M. van der Sman, "Optimal Adaptive Scheduling and Control of Beer Membrane Filtration", Control Engineering Practice, Vol. 34, pp. 77-87, 2015.

[25] S. Duranceau, "Membrane Practices for Water Treatment", American Water Works Association, 2001.

[26] C. M. Chew, M. K. Aroua, M. A. Hussain, and W. M. Ismail, "Evaluation of Ultrafiltration and Conventional Water Treatment Systems for Sustainable Development: An Industrial Scale Case Study", Journal of Cleaner Production, Vol. 112, pp. 3152-3163, 2016.

[27] S. Jiménez, M.M. Micó, M. Arnaldos, F. Medina, and S. Contreras, "State of the Art of Produced Water Treatment", Chemosphere, Vol. 192, pp. 186-208, 2018.

[28]V. G. Gude, "Energy and Water Autarky of Wastewater Treatment and Power Generation Systems", Renewable and Sustainable Energy Reviews, Vol. 45, pp. 52-68, 2015.

[29]J. R.Wolfe, R. A. Goldstein, J. S. Maulbetsch, and C. R. McGowin, "An Electric Power Industry Perspective on Water Use Efficiency", Journal of Contemporary Water Research \& Education, Vol. 143, pp. 30-34, 2009.

[30]W. Shao, J. Feng, J. Liu, G. Yang, Z. Yang, and J. Wang, "Research on the Status of Water Conservation in the Thermal Power Industry in 
China", Energy Procedia, Vol. 105, pp. 30683074, 2017. 